\title{
Realistic model of a vertical pillar quantum dot: Analysis of individual dot data
}

\author{
P. A. Maksym, ${ }^{1}$ Y. Nishi, ${ }^{2}$ D. G. Austing, ${ }^{3}$ T. Hatano,${ }^{4}$ L. P. Kouwenhoven,${ }^{5}$ H. Aoki,${ }^{2}$ and S. Tarucha $a^{4,6}$ \\ ${ }^{1}$ Department of Physics and Astronomy, University of Leicester, Leicester LE1 7RH, United Kingdom \\ ${ }^{2}$ Department of Physics, University of Tokyo, Hongo, Bunkyo-ku, Tokyo 113-0033, Japan \\ ${ }^{3}$ Institute for Microstructural Sciences M50, National Research Council, Montreal Road, Ottawa, Ontario, Canada K1A OR6 \\ ${ }^{4}$ ICORP Spin Information Project, JST, Atsugi-shi, Kanagawa 243-0198, Japan \\ ${ }^{5}$ Department of Applied Physics, Delft University of Technology, P.O. Box 5046, 2600 GA Delft, The Netherlands \\ ${ }^{6}$ Department of Applied Physics, University of Tokyo, Hongo, Bunkyo-ku, Tokyo 113-8656, Japan \\ (Received 20 April 2008; revised manuscript received 10 February 2009; published 23 March 2009)
}

\begin{abstract}
An accurate model of a vertical pillar quantum dot is described. The full three-dimensional structure of the device containing the dot is taken into account and this leads to an effective two-dimensional model in which electrons move in the two lateral dimensions, the confinement is parabolic, and the interaction potential is very different from the bare Coulomb potential. The potentials are found from the device structure and a few adjustable parameters. Numerically stable calculation procedures for the interaction potential are detailed and procedures for deriving parameter values from experimental addition energy and chemical potential data are described. The model is able to explain magnetic-field-dependent addition energy and chemical potential data for an individual dot to an accuracy of about 5\%, the accuracy level needed to determine ground-state quantum numbers from experimental transport data. Applications to excited state transport data are also described.
\end{abstract}

DOI: 10.1103/PhysRevB.79.115314

PACS number(s): 73.63.Kv, 73.23.Hk

\section{INTRODUCTION}

Vertical pillar quantum dots exhibit an extremely wide range of interesting physics, particularly in the presence of a magnetic field perpendicular to the plane of the dot. ${ }^{1-3}$ The Coulomb blockade allows single electrons to be added to the dot so the number of electrons can be set precisely, starting from one. For each number of electrons the ground state evolves through a series of transitions as the magnetic field is increased. At zero magnetic field the ground states are well described by Hund's first rule and maximum density droplet (MDD) states occur when the field is increased to a few Tesla. Finally, fractional quantum Hall droplet and electron molecule states occur at high fields beyond about $10 \mathrm{~T}$. So, roughly speaking, there are four regimes but the detailed picture is much richer. Many transitions occur in between each of the main regimes and each transition is accompanied by abrupt changes in the spin and orbital angular momentum of the ground state. There is experimental ${ }^{1-5}$ and theoretical ${ }^{5-10}$ evidence that these effects occur. However it is difficult to apply experimental techniques, such as scanning tunneling spectroscopy, to probe the corresponding quantum states directly. Instead the ground-state quantum numbers are found by comparing data from transport spectroscopy with calculated results. This requires an accurate dot model that can be used to analyze data for an individual quantum dot. Development of an appropriate model is the purpose of the present work.

Although quantum dots are often described as artificial atoms, there is a very important difference between an artificial atom and a natural one: all natural atoms of the same isotope are identical but all quantum dots are different. This makes it quite challenging to model an individual dot accurately, even when its basic design parameters are known, because manufacturing tolerances introduce fluctuations in the parameters and this can have a significant influence on dot states. The fact that the dot often consists of a small region embedded in a much larger device structure just adds to the difficulty of constructing an accurate model. The ideal model of an electrostatic quantum dot is a system in which electrons are constrained to move in the two dimensions parallel to the dot plane, are confined by a parabolic potential, and interact via the Coulomb interaction. The properties of this model have been examined extensively; see Refs. 10 and 11 for reviews. It gives a good qualitative description of dot behavior but a more accurate model is needed for data analysis. One possibility is a device model in which the dot confining potential and in some cases the interaction potential are computed, together with the quantum states, from a combined solution of the Poisson and Schrödinger equations. This approach was first used to compute the states of dots defined by a modulated gate ${ }^{12}$ and was subsequently applied to dots of the vertical pillar type, ${ }^{13-16}$ for example. It has given a great deal of insight into the generic behavior of devices containing quantum dots. However, it is extremely difficult to use generic device models to deduce ground-state quantum numbers from experimental data for an individual quantum dot.

One of the difficulties encountered in analyzing individual dot data is that energies have to be computed to very high precision. The quantity measured experimentally is the gate voltage at which transport occurs. This is proportional to the chemical potential, $\mu_{N}$, which is the difference of two dot energies: $\mu_{N}=E_{N}-E_{N-1}$, where $E_{N}$ is the energy of the an $N$-electron dot state. Further, the transport data are often presented as a difference between the gate voltages for two successive current peaks. This gives the addition energy, $E_{A N}=E_{N+1}-2 E_{N}+E_{N-1}$, the second difference of $E_{N}$ with respect to $N$. Because energy differences are needed to compare theoretical results with experimental data the energies themselves must be computed very accurately. Typically, the addition energy is around $3 \mathrm{meV}$ while typical dot energies are 1 or 2 orders of magnitude larger so that high precision 


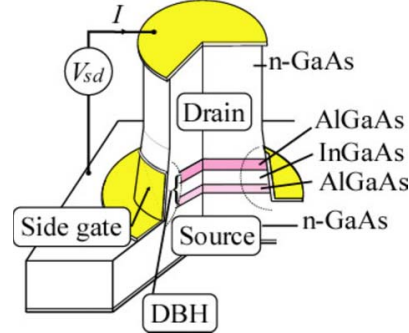

(a)

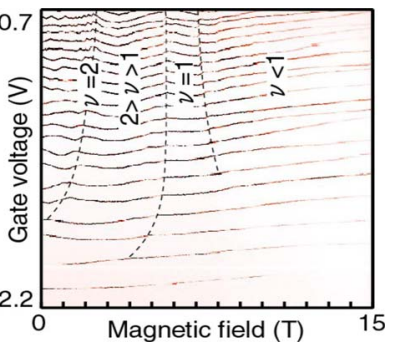

(b)

FIG. 1. (Color online) (a) Typical vertical pillar dot and (b) typical transport data. Labels indicate the Landau level filling factor $\nu$.

values of the dot energy are needed for data analysis. However the potentials found in generic dot models depend on many parameters, dot dimensions, dopant densities, etc., some of which are not known accurately. In principle, these parameters could be adjusted to fit the data but it is desirable to avoid fitting a large number of parameters.

The present model lies between the parabolic confinement, Coulomb interaction model, and the generic device models. The main idea is to develop a model that contains all the essential physics but depends on a small number of adjustable parameters. Three are used in the present work but it turns out that one of them is not very significant. The model is a parabolic confinement model, believed to be accurate for small numbers of electrons. ${ }^{13}$ Two of the parameters determine the parabolic potential but only one of them is significant. The interaction potential is determined from a simplified device structure. The effects of finite thickness and screening on the interaction in this structure are included fully and a third model parameter is used to determine the screening. Essentially, the resulting model reduces to a twodimensional, parabolic confinement model with an interaction potential that is very different from the bare Coulomb potential. The model gives an excellent description of addition energy data, accurate to about $0.15 \mathrm{meV}$. It has already been used to determine the ground-state spin and orbital angular momentum of a pillar dot in the strong magnetic-field regime. ${ }^{5}$ However Ref. 5 does not contain the detailed explanation of the model and analysis procedures that is given here.

The model is described in Sec. II and the method used to calculate the quantum states is detailed in Sec. III. Following this, parameter fitting procedures for experimental addition energy and chemical potential data are discussed in Sec. IV. Then the application of the model to data in the low and high magnetic-field regimes is described in Sec. V and conclusions are stated in Sec. VI. Finally, the Appendix details numerical procedures.

\section{DOT MODEL}

\section{A. Vertical pillar dots}

A vertical pillar dot [Fig. 1(a)] consists of a narrow pillar, typically $500 \mathrm{~nm}$ in diameter. ${ }^{4}$ The pillar contains a double barrier heterostructure $(\mathrm{DBH})$ which provides confinement in the vertical $(z)$ direction and is surrounded by a cylindrical metal gate which carries a negative charge that provides lateral $(x, y)$ confinement. The pillar stands on a substrate which is heavily doped and there is also a heavily doped region at the top of the pillar. These heavily doped regions provide source and drain contacts that enable the transport properties of the device to be investigated. A unique advantage of the vertical geometry is that it is not sensitive to edge states which affect transport through lateral devices in the high magnetic-field regime.

In a typical experiment, the source-drain current is measured as a function of the gate voltage $V_{g}$, source-drain voltage $V_{s d}$, and magnetic field $B$ parallel to the pillar. Typical results ${ }^{5}$ are shown in the bottom frame of Fig. 1. This image shows the condition for an electron to enter the dot at fixed source-drain bias. It is well known that transport through the device is Coulomb blockaded except at certain values of $V_{g}$ and $V_{s d}$ (Ref. 4). In Fig. 1 the current is intensity coded so that dark regions correspond to the largest current. The $N$ th dark curve shows how the gate voltage needed for the $N$ th electron to enter the dot depends on the magnetic field. The lowest curve in the figure corresponds to $N=1$.

The exact condition for electron transport is

$$
\mu_{c}+\frac{e V_{s d}}{2} \geq \mu_{N}-e \alpha\left(V_{g N}\right) V_{g N} \geq \mu_{c}-\frac{e V_{s d}}{2},
$$

where $\mu_{c}$ is the contact chemical potential, $V_{g N}$ is the gate voltage at which the $N$ th electron enters the dot, and $\alpha$ is an electrostatic leverage factor. At zero source-drain bias this reduces to

$$
e \alpha\left(V_{g N}\right) V_{g N}=\mu_{N}-\mu_{c}
$$

so that the gate voltage at which transport occurs is a measure of the dot chemical potential relative to the contact. For transport data in the form of a gate voltage difference, $V_{g N}-V_{g N-1}$ is proportional to the addition energy, $E_{A N}=E_{N+1}-2 E_{N}+E_{N-1}$ if $\alpha$ and $\mu_{c}$ are independent of $N$. Hence the first and second differences of the dot energies are needed to compare theory with experiment.

\section{B. Overview of model}

The dot model takes account of vertical confinement, lateral confinement, and interactions between dot electrons. The quantum well between the double barriers is relatively narrow $(12 \mathrm{~nm})$ so the electrons are confined to the ground state of the vertical motion. This leads to a quasi-two-dimensional model in which the interaction is modified by the vertical confinement, physically the effect is to smear out the Coulomb singularity.

The lateral confinement is generated by the cylindrical side gate and in principle the lateral confinement potential may be found by solving the Poisson equation, Refs. 12-15 and 17 , for example. However in the present case, $N$ is small so the spatial extent of the dot state $(<50 \mathrm{~nm})$ is very small compared to the pillar diameter $(\approx 500 \mathrm{~nm})$. Therefore the only part of the confining potential that has a significant effect on the dot electrons is the part near the minimum which is parabolic. In addition, observation of shell structure 
in the device considered here ${ }^{5}$ shows that the device has a high degree of circular symmetry. The lateral confining potential is therefore taken to be $V_{c}=V_{0}+m^{*} \omega_{0}^{2} r^{2} / 2$, where $r$ is the lateral radial coordinate and $\omega_{0}$ is found from $\hbar \omega_{0}=a+b(N-1)$, where $a$ and $b$ are fitting parameters. The constant $V_{0}$ is not needed for the present analysis because the addition energy is independent of $V_{0}$ and the chemical potential is also independent of $V_{0}$ when $\mu_{N}$ taken relative to $\mu_{1}$. Previous work has shown that the parabolic approximation is consistent with experiment ${ }^{6}$ and accurate for small numbers of electrons, ${ }^{13}$ that is, electrons in the first and second shells at $B=0$.

The screening is mainly caused by heavily doped contacts that are relatively close to the dot, around $10 \mathrm{~nm}$ from the well. This is close enough for the contact regions to have a significant screening effect on the interaction between the dot electrons. This is treated in the Thomas-Fermi approximation and the screening length in the contact region is taken to be a fitting parameter. Full details of the present approach to screening and finite thickness are given in the following two sections.

\section{Finite thickness}

The finite thickness is taken into account via the envelope function for electrons in the ground state of the vertical quantum well. In addition to the smearing of the Coulomb singularity, this also changes the effective mass to the average mass, $\bar{m}^{*}$ given by

$$
\frac{1}{\bar{m}^{*}}=\frac{p_{w}}{m_{w}^{*}}+\frac{\left(1-p_{w}\right)}{m_{b}^{*}},
$$

where $p_{w}$ is the probability of finding an electron in the well. The dot considered here is made from InGaAs containing 5\% In, and the In reduces the mass. The barrier is made from AlGaAs containing $22 \% \mathrm{Al}$ and this increases the mass. The net effect is to reduce the mass to $0.0653 m_{0}$, slightly less than the GaAs value. The averaging procedure also applies to systems with spin-orbit coupling. In this case, it is found that the Dresselhaus spin-orbit coupling parameter has to be averaged in a way similar to the effective mass. This has been used for studies of spin relaxation in the present device. ${ }^{18}$

The finite thickness also contributes to the effective $g$ factor, $\left|g^{*}\right|$, for the dot which is very different from the bulk effective $g$ factor. The dot $g$ factor is determined from the experimentally observed Zeeman splitting 5 in the same device that is used for the data analysis performed here. $\left|g^{*}\right|=0.3$ for the dot when $B \gtrsim 10 \mathrm{~T}$, compared with $\left|g^{*}\right|=0.44$ for quasi-2D GaAs. The reduced value for the dot is consistent with the effect of vertical and lateral nonparabolicity. ${ }^{19}$ The nonparabolicity also makes the effective $g$ factor depend on magnetic field and Landau level index ${ }^{19}$ but the resulting corrections to $\mu_{N}$ are probably smaller than experimental error. A constant $g$ factor is therefore used and the Zeeman energy is accounted for by adding $g^{*} \mu_{B} B S_{z}$ to the orbital energy. Here $\mu_{B}$ is the Bohr magneton and $S_{z}$ is the $z$ component of the total spin $S$. The effective $g$ factor is assumed to be negative, as in bulk GaAs, so $g^{*}=-0.3$.

\section{Screening}

The most important source of screening in the present device is the heavily doped contacts above and below the dot. The dielectric response of the disordered contact material is not well understood but can be treated approximately in the static Thomas-Fermi approximation. This allows the screening length to be estimated from the density of states at the Fermi level, however, this is not known accurately for the contact material. Hence the screening length is taken to be a fitting parameter which is determined from experimental data.

The screened interaction between dot electrons ${ }^{20}$ is found from the electrostatic Green's function $G\left(\mathbf{R}, \mathbf{R}^{\prime}\right)$ which satisfies

$$
\begin{aligned}
-\nabla \cdot\left[\left(\epsilon(\mathbf{R}) \epsilon_{0} \nabla G\left(\mathbf{R}, \mathbf{R}^{\prime}\right)\right]=\right. & -q_{0}^{2}(\mathbf{R}) \epsilon(\mathbf{R}) \epsilon_{0} G\left(\mathbf{R}, \mathbf{R}^{\prime}\right) \\
& +\delta\left(\mathbf{R}-\mathbf{R}^{\prime}\right),
\end{aligned}
$$

where $\epsilon(\mathbf{R})$ is the relative permittivity, $q_{0}=2 \pi / \lambda_{s}$ inside the contacts, $q_{0}=0$ outside the contacts, and $\mathbf{R}=(\mathbf{r}, z)$. Because a detailed theory of magnetic-field-dependent screening by the three-dimensional (3D) contact material is not available, the screening length, $\lambda_{s}$, is taken to be independent of the magnetic field. This approximation should be good when the screening length does not change significantly over the field range used in the experiments and it is assumed that this is the case. Equation (4) is simplified by making use of the fact that the dot is an order of magnitude smaller than the pillar. This means that the Green's function is almost translationally invariant in the lateral direction and can be found to a good approximation by replacing the pillar by a stack of dielectric layers of infinite extent in the lateral directions. $\epsilon$ and $q_{0}$ then do not depend on $\mathbf{r}$. So the Green's function is translationally invariant in the lateral directions and can be expressed as a Fourier transform:

$$
G\left(\mathbf{R}, \mathbf{R}^{\prime}\right)=\frac{1}{4 \pi^{2}} \int G\left(q, z, z^{\prime}\right) \exp \left[i \mathbf{q} \cdot\left(\mathbf{r}-\mathbf{r}^{\prime}\right)\right] d \mathbf{q} .
$$

The Fourier components of the Green's function satisfy

$$
\begin{aligned}
& -\frac{d}{d z}\left[\epsilon(z) \epsilon_{0} \frac{d}{d z} G\left(q, z, z^{\prime}\right)\right]+\epsilon(z) \epsilon_{0}\left[q^{2}+q_{0}^{2}(z)\right] G\left(q, z, z^{\prime}\right) \\
& =\delta\left(z-z^{\prime}\right) .
\end{aligned}
$$

The solution of this equation has the form

$$
\begin{array}{ll}
G\left(q, z, z^{\prime}\right)=-\frac{f(z) g\left(z^{\prime}\right)}{W}, & z>z^{\prime}, \\
G\left(q, z, z^{\prime}\right)=-\frac{g(z) f\left(z^{\prime}\right)}{W}, & z<z^{\prime},
\end{array}
$$

where $f$ and $g$ are solutions of the homogeneous equation corresponding to Eq. (6) and $W$ is their Wronskian. These solutions are chosen to satisfy $f \rightarrow 0$ when $z \rightarrow+\infty$ and $g \rightarrow 0$ when $z \rightarrow-\infty$. Although the analytic form of $G$ is as given in Eq. (7), severe numerical instabilities are encountered when this form is evaluated unless special precautions are taken. The problem is that the $G$ has rapidly growing 


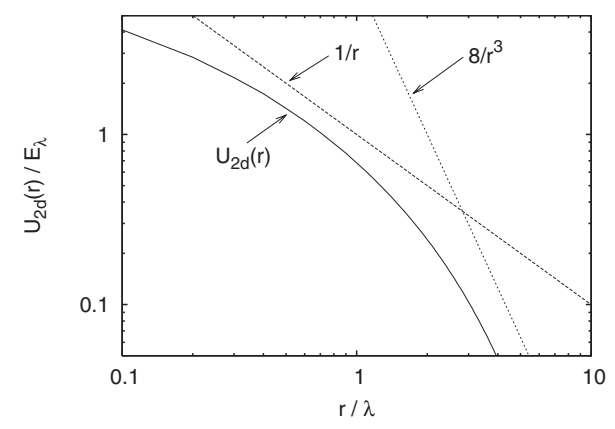

FIG. 2. Effective 2D interaction (solid line). Dashed lines show the $1 / r^{3}$ limiting form and the $1 / r$ Coulomb interaction.

components which cause exponent overflow. Exactly the same mathematical problem is encountered in calculations of evanescent wave propagation in electron diffraction theory and the solution used here is a reflection matrix approach developed for calculations of reflection high-energy electron diffraction. ${ }^{21-23}$ This is detailed in the Appendix.

Once the Green's function has been found, the Fourier components of the effective two-dimensional interaction are obtained in the form of an integral:

$$
U_{2 d}(q)=e^{2} \int \chi^{2}(z) \chi^{2}\left(z^{\prime}\right) G\left(q, z, z^{\prime}\right) d z d z^{\prime},
$$

where $\chi$ is the quantum well ground state. The separable form of the Green's function, Eq. (7), enables this integral to be evaluated efficiently. The form of the effective interaction in real space is very different from a pure Coulomb interaction. Because of the screening, the interaction is dipolelike at long range and decreases like $1 / r^{3}$ in the limit of large $r$. Also the Coulomb singularity is removed by the finite thickness because the electrons are able to move out of the dot plane and avoid each other. The real-space effective interaction as a function of $r$ is shown in Fig. 2. $U_{2 d}(r)$ is calculated numerically from its Fourier transform $U_{2 d}(q)$ for the layer structure shown in Fig. 1 (see also Table I) and $\lambda_{s}=10 \mathrm{~nm}$, a typical screening length. The length unit is the $2 \mathrm{D}$ harmonic oscillator length parameter, $\lambda^{2}=\hbar /\left(2 m^{*} \Omega\right)$ where $\Omega^{2}=\omega_{0}^{2}+\omega_{c}^{2} / 4$ with $\omega_{c}$ as the cyclotron frequency. The energy unit $E_{\lambda}=e^{2} /\left(4 \pi \epsilon_{w} \epsilon_{0} \lambda\right)$ where $\epsilon_{w}$ is the relative permittivity in the well that contains the dot. It is clear that $U(r)$ is significantly different from the $1 / r$ Coulomb interaction and

TABLE I. Layer structure of the device model.

\begin{tabular}{lcc}
\hline \hline Layer & Composition & $\begin{array}{c}\text { Thickness } \\
(\mathrm{nm})\end{array}$ \\
\hline Top contact & $n^{+} \mathrm{GaAs}$ & \\
Buffer & $i$ GaAs & 3 \\
Barrier & $\mathrm{Al}_{0.22} \mathrm{Ga}_{0.78} \mathrm{As}$ & 9 \\
Well & $\mathrm{In}_{0.05} \mathrm{Ga}_{0.95} \mathrm{As}$ & 12 \\
Barrier & $\mathrm{Al}_{0.22} \mathrm{Ga}_{0.78} \mathrm{As}$ & 7.5 \\
Buffer & $i \mathrm{GaAs}$ & 3 \\
Bottom contact & $n^{+} \mathrm{GaAs}$ & \\
\hline
\end{tabular}

approaches the $1 / r^{3}$ form in the limit of large $r$. The same limiting form appears in other quasi-2D systems. ${ }^{24}$

The effect of the dielectric interfaces in the device on the effective interaction is accounted for fully in the Green's function formalism. In addition, the dielectric interfaces have an effect on the vertical confinement potential. This results from the interaction of each electron with its own image charges and is also accounted for in the Green's function formalism. The image charge contribution to the vertical confinement is obtained from the nonsingular part of the Green's function ${ }^{20}$ and in the present case the image charge contribution is

$$
V_{I}(z)=\frac{e^{2}}{8 \pi \epsilon_{w} \epsilon_{0}} \int_{0}^{\infty}\left[2 q \epsilon_{w} \epsilon_{0} G(q, z, z)-\frac{\epsilon_{w}}{\widetilde{\epsilon}}\right] d q,
$$

where $\tilde{\boldsymbol{\epsilon}}=\epsilon_{w}$ when $z$ is in the well, $\tilde{\boldsymbol{\epsilon}}=\epsilon_{b}$, the barrier relative permittivity, when $z$ is in the barrier and $\widetilde{\epsilon}=\left(\epsilon_{w}+\epsilon_{b}\right) / 2$ when $z$ is at the interface between the well and the barrier. The image charge term leads to a small change in the vertical confinement which is taken into account via perturbation theory.

Another source of screening in the present device is the metallic, cylindrical side gate. In principle, this breaks the translational invariance of the effective lateral interaction however this effect is expected to be small. The magnitude of the effect can be estimated from the electrostatic Green's function for an infinite metallic cylinder. ${ }^{25}$ Numerical calculations of the Green's function suggest that the effect is $\lesssim 1 \%$ within a region of radius $\sim 10 \mathrm{~nm}$ in the center of the cylinder. The effect is clearly largest for electrons at the edge of the dot but the interaction between these electrons and electrons in the center of the dot is suppressed by the screening effect of the contacts. These considerations suggest that the effect of the gate is small for the small electron numbers considered here $(N \leq 5)$. However, the effect could be significant for larger electron droplets whose edge is closer to the metallic gate.

\section{E. Model Hamiltonian}

The effective Hamiltonian for the two-dimensional dot model is

$$
H_{\mathrm{eff}}=\sum_{i} \frac{\pi_{i}^{2}}{2 \bar{m}^{*}}+V_{c}\left(r_{i}\right)+N E_{z}+g^{*} \mu_{B} B S_{z}+\frac{1}{2} \sum_{i \neq j} U_{2 d}\left(\mathbf{r}_{i}-\mathbf{r}_{j}\right),
$$

where $\boldsymbol{\pi}=\mathbf{p}_{\|}+e \mathbf{A}, \mathbf{p}_{\|}$is the lateral momentum, $\mathbf{A}$ is the magnetic vector potential, $\mathbf{r}=(x, y), V_{c}$ is the lateral confining potential, $E_{z}=\left\langle\chi\left|H_{\perp}\right| \chi\right\rangle+\Delta V_{I}$ is the perpendicular energy, and $\Delta V_{I}$ is the perturbation caused by the image charge term. The effective $g$ factor is determined experimentally (Sec. II C). The layer structure of the device is detailed in Table I and the material parameters used for the present calculations are detailed in Table II. Although the model Hamiltonian has been justified here on physical grounds it can also be justified formally by considering a variational trial function of form $\Psi=\Pi_{i} \chi\left(z_{i}\right) \Phi\left(\mathbf{r}_{1}, \ldots \mathbf{r}_{N}\right)$, where $\Phi$ is antisymmetric and includes spin functions. When this is used to minimize the 
TABLE II. Material parameters for the device model.

\begin{tabular}{lcc}
\hline \hline Parameter & Material & Value \\
\hline$m^{*} / m_{0}$ & $\mathrm{Al}_{x} \mathrm{Ga}_{1-x} \mathrm{As}$ & $0.067+0.083 x$ \\
$m^{*} / m_{0}$ & $\mathrm{In}_{y} \mathrm{Ga}_{1-y} \mathrm{As}$ & $0.067-0.045 y$ \\
$V_{b}(\mathrm{meV})$ & $\mathrm{Al}_{x} \mathrm{Ga}_{1-x} \mathrm{As}_{2} \mathrm{In}_{y} \mathrm{Ga}_{1-y} \mathrm{As}$ & $823 x+640 y$ \\
$\epsilon_{b}$ & $\mathrm{Al}_{x} \mathrm{Ga}_{1-x} \mathrm{As}$ & $12.7-3.12 x$ \\
$\epsilon_{w}$ & $\mathrm{In}_{y} \mathrm{Ga}_{1-y} \mathrm{As}$ & $12.7+2 y$ \\
\hline \hline
\end{tabular}

expectation value of the full 3D Hamiltonian, the result is $H_{\text {eff }}$ plus small corrections, with the effective mass given by Eq. (3).

\section{CALCULATION OF EIGENSTATES}

The effective Hamiltonian $H_{\text {eff }}$ is very similar to the Hamiltonian for a two-dimensional parabolic dot with a Coulomb interaction. Hence the eigenstates of $H_{\text {eff }}$ are found in the usual way by numerical diagonalization in a FockDarwin basis. ${ }^{10}$ The only difference between the usual calculation and the present one is that matrix elements of the effective interaction $U_{2 d}$ have to be computed. In the case of the Coulomb interaction these matrix elements are normally found from the Fourier transform of the interaction and the calculation involves an integral over $q$ (Ref. 10). In the present case the Fourier transform approach is also used. $U_{2 d}(q)$ has the form $F(q) V_{2 d}(q)$, where $V_{2 d}(q)$ is the Fourier transform of the Coulomb interaction, $e^{2} /\left(4 \pi \epsilon_{w} \epsilon_{0} r\right)$, in two dimensions, and $F(q)$ is a form factor that is computed numerically from the Green's function. The only difference between the standard treatment of the interaction in a $2 \mathrm{D}$ dot and the present treatment is the appearance of the form factor in the $q$ integral. This integral is done numerically by Romberg integration.

The Fock-Darwin states are labeled by an angular momentum quantum number $l$ and a radial quantum number $n$. The many-electron basis used for the diagonalization consists of Slater determinants formed from these states. It is important to minimize the size of this basis as expensive calculations have to be performed repeatedly to fit the model parameters. The Hamiltonian is block diagonalized according to the value of the total orbital angular momentum $L$ and total spin $S$. For $N \leq 4$ the basis for each $L$ is formed from Fock-Darwin states with $n \leq 3$ and all $l$ values compatible with the required $L$ value. For $N=5$ the size of the basis is limited by making use of the fact that the radial excitation is an inter-Landau level excitation and hence has large energy in a strong magnetic field. This enables the maximum $n$ quantum number to be reduced as a function of magnetic field. In the present case, the maximum value of $n$ is taken to be the integer part of $6.9-0.28 B$ which corresponds to a maximum $n$ of 6 at $B=0 \mathrm{~T}$ and 2 at $B=14 \mathrm{~T}$. All Slater determinants compatible with this $n$ value are then constructed and those Slater determinants whose energy is within $100 \mathrm{meV}$ of the lowest energy determinant are retained in the calculation. This rejection step reduces the total number of determinants slightly and saves some computer time. The accuracy of the numerically calculated groundstate addition energies is estimated to be $0.1 \mathrm{meV}$ or better. The $B$-dependent cutoff on $n$ leads to small steps in the energy as a function of $B$ which result from the sudden change in the number of basis states that occurs whenever the integer part of $6.9-0.28 B$ changes. These artifacts are typically around $0.01 \mathrm{meV}$, about an order of magnitude smaller than experimental error.

\section{DATA ANALYSIS}

\section{A. Parameter fitting}

The model parameters are obtained from an unweighted least-squares fit of the entire data set. This corresponds to minimizing the rms difference between experiment and theory. The minimization is done with the LevenbergMarquardt algorithm. ${ }^{26}$ This procedure is generally accepted to give reliable parameter values provided the number of features in the data exceeds the number of fitting parameters, a condition that is satisfied in the present case.

\section{B. Experimental data}

Two data sets are used. The first (set 1) was collected as part of an experiment to investigate ground-state transitions in the high-magnetic field regime. ${ }^{5}$ These data are used to analyze addition energies (Figs. 3 and 4). The data were taken with a fairly high magnetic-field resolution $(0.05 \mathrm{~T})$ and consists of a limited number of $V_{g N}$ curves, $N=2,3,4$, and 5 only. The measurements were performed in a dilution refrigerator and the electron temperature is estimated to be below $100 \mathrm{mK}$.

The second data set (set 2) is used to analyze chemical potentials (Fig. 5). These data were collected under similar conditions to set 1 but over a wider range of $N$, a smaller magnetic-field range and a lower field resolution $(0.1 \mathrm{~T})$. In addition, the device was subjected to one thermal cycle between collecting the two data sets. Set 2 was collected first then the device was taken up to room temperature and cooled again to collect set 1 .

\section{Analysis of addition energy data}

The ground-state addition energy is the difference of two successive chemical potentials: $E_{A N}=E_{N+1}-2 E_{N}+E_{N-1}$ $=\mu_{N+1}-\mu_{N}$. It follows from Eq. (2) that $E_{A N}$ at fixed magnetic field is related to two successive gate voltages:

$$
E_{A N}=e\left[\alpha\left(V_{g N+1}\right) V_{g N+1}-\alpha\left(V_{g N}\right) V_{g N}\right],
$$

which is valid provided that the contact chemical potential $\mu_{c}$ is independent of $V_{g}$ and therefore cancels when the $\alpha V_{g}$ values are subtracted. Equation (11) can in principle be used to find $E_{A N}$ from experimental values of $\alpha$ and $V_{g}$. However this requires very accurate values of both $\alpha$ and $V_{g}$. Typical values of $E_{A N}$ are around $3 \mathrm{meV}$, while typical values of $e \alpha V_{g}$ are around $60 \mathrm{meV}$. So both $\alpha$ and $V_{g}$ need to be measured to an accuracy of $0.1 \%$ or better to measure $E_{A N}$ to $\pm 0.1 \mathrm{meV}$. $V_{g}$ is known to this accuracy for the present 


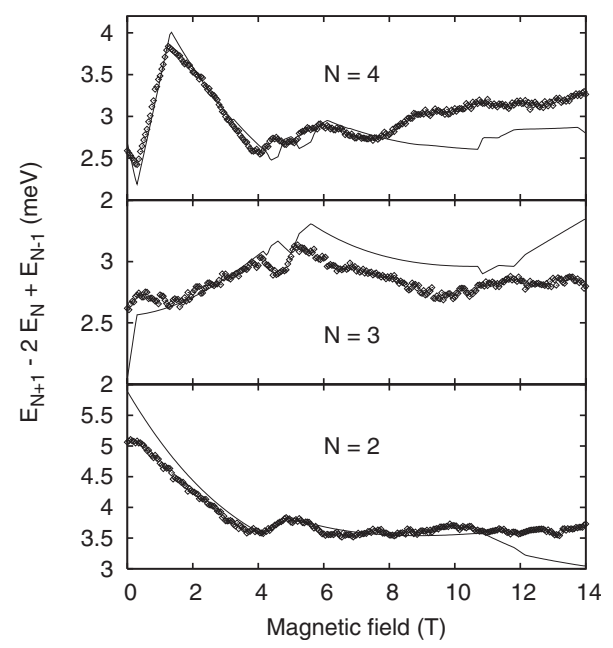

FIG. 3. Experimental and theoretical addition energies. The field range used for parameter fitting is $0 \leq B \leq 14 \mathrm{~T}$. Diamonds: experimental data, solid lines: theoretical results.

device but $\alpha$ is not. Equation (11) is therefore approximated by

$$
E_{A N}=e \bar{\alpha}_{N+1}\left(V_{g N+1}-V_{g N}\right),
$$

where $\bar{\alpha}_{N+1}=\left[\alpha\left(V_{g N+1}\right)+\alpha\left(V_{g N}\right)\right] / 2$. This suppresses the random errors which would result from use of Eq. (11) but is only valid when $\alpha\left(V_{g N}\right)$ varies sufficiently slowly with $N$. This is assumed to be the case. The validity of this assumption cannot be tested without more data on $\alpha$ however the results based on this assumption are found to be consistent with results of chemical potential analysis, Sec. IV D. Another factor that limits the present analysis is that $\alpha$ depends on the magnetic field as well as $V_{g}$ but is only known for a sample of field points at intervals of around 1-2 T. The $\alpha$ values at intermediate field points have to be obtained by linear interpolation and this introduces a further systematic error which is difficult to quantify. In principle, both this systematic error and the one resulting from use of $\bar{\alpha}$ can be eliminated by performing detailed measurements of $\alpha$.

Figure 3 shows the comparison between experimental and theoretical addition energies for $N=2,3$, and 4 . All three curves were used simultaneously to fit the parameters, as described in Sec. IV A. It is clear that the absolute values of the experimental and theoretical data agree very well. The raw experimental data are shown in the figure together with raw theoretical data. There is no shifting or scaling of the theoretical curves. The agreement is generally good but there are some discrepancies, particularly around 4-6 T and above $10 \mathrm{~T}$. To investigate the cause of these discrepancies the data were fitted again in the restricted field range $0 \leq B \leq 10 \mathrm{~T}$. The results are shown in Fig. 4 and it is clear that the fit is better, particularly around 4-6 $\mathrm{T}$ where the double peak structure for $N=3$ and $N=4$ is reproduced very well. The improvement in the fit can be quantified by the rms difference between experiment and theory. The value corresponding to Fig. 3 is $0.23 \mathrm{meV}$ while for Fig. 4 it is $0.15 \mathrm{meV}$.

The deterioration in the quality of the fit when the full data range is used could be caused by factors not included in

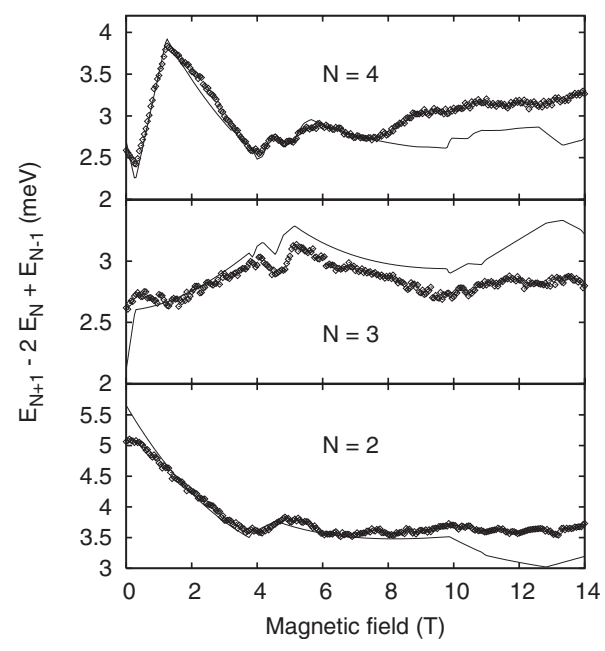

FIG. 4. Experimental and theoretical addition energies. As Fig. 3 except that the field range used for parameter fitting is $0 \leq B \leq 10 \mathrm{~T}$.

the model or uncertainties in the experimental data. The most likely causes are impurity effects, screening, and insufficient knowledge of $\alpha$.

Both impurity effects and screening could depend on magnetic field. Impurity effects are not included in the present model but are likely to be more significant in the very strong field regime. The mean distance between the impurities in the heavily doped contacts is around $10 \mathrm{~nm}$. This gives a fluctuating contribution to the dot potential on a similar length scale. But at zero magnetic field the typical diameter of the dot state is around $20-30 \mathrm{~nm}$. Hence the effect of the impurities will tend to average out. Indeed the experimental evidence is that the present device has a high degree of circular symmetry. ${ }^{5}$ However as the magnetic field increases the dot wave function shrinks and eventually becomes smaller than the length scale of the potential fluctuations. Impurity effects would be large in this regime but the magnetic fields used in the present work are probably too low for this to happen. Magnetic-field-dependent screening is another possible cause of discrepancies but if the magnetic-field dependence was significant, it would probably occur throughout the field range, while the discrepancies are mainly in the high field range.

The remaining possible cause of the discrepancies is that the value of $\alpha$ is not known to sufficient accuracy in the

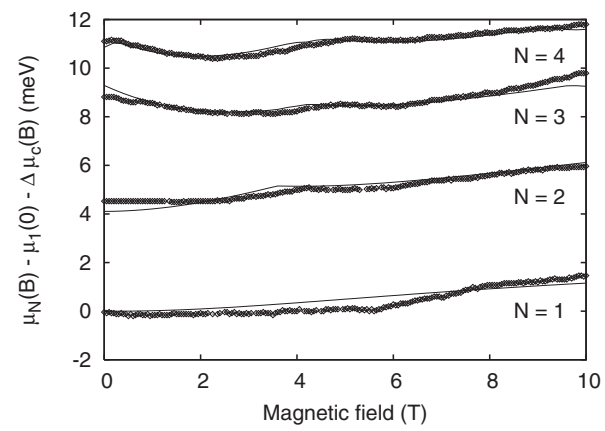

FIG. 5. Experimental and theoretical chemical potentials. Diamonds: experimental data, solid lines: theoretical results. 
high-field regime. In this case the parameters determined by fitting the data up to $10 \mathrm{~T}$ are expected to provide an accurate description of the dot in the field range up to $14 \mathrm{~T}$ but the problem lies in the numerical value of the experimental addition energy. There is some evidence that this is the case: excited state features predicted by the present model are found to correspond to features in experimental excitation spectra in the high-field regime. ${ }^{5}$ The value of $\alpha$ is not needed to identify these features and this suggests that the discrepancies in the high field addition energy are most likely to be related to insufficient knowledge of $\alpha$.

The best fit parameter values corresponding to Fig. 4 are $a=4.8 \pm 0.1 \mathrm{meV}, \quad b=0.02 \pm 0.01 \mathrm{meV}, \quad$ and $\lambda_{s}$ $=15.0 \pm 3 \mathrm{~nm}$. Here the statistical errors correspond to the parameter range compatible with the fluctuations in the data shown in Fig. 4 but exclude the unquantifiable uncertainty in $\alpha$. These values are taken to give the best model of the present device. The value of $b$ suggests that the confinement energy increases slowly with gate voltage but the opposite would be expected from electrostatics of the device. However the value of $b$ is comparable to its error so a model with constant confinement or slowly decreasing confinement would probably give an equally good fit to the data.

\section{Analysis of chemical potential data}

In principle, Eq. (2) can be used to obtain $\mu_{N}$ from the $V_{g}$ data but the appearance of the contact chemical potential $\mu_{c}$ in this equation presents an obstacle. The absolute value of $\mu_{c}$ is not known. In addition, $\mu_{c}$ is expected to vary with magnetic field and its field dependence is not known. Part of the difficulty can be eliminated by taking the chemical potential relative to the chemical potential for the first electron at zero magnetic field. The contact chemical potential is written as $\mu_{c}(0)+\Delta \mu_{c}(B)$, where $\Delta \mu_{c}$ vanishes when $B=0$. Equation (2) becomes

$$
\begin{gathered}
e\left[\alpha\left(V_{g N}, B\right) V_{g N}(B)-\alpha\left(V_{g 1}, 0\right) V_{g 1}(0)\right] \\
=\mu_{N}(B)-\mu_{1}(0)-\Delta \mu_{c}(B) .
\end{gathered}
$$

This enables experimental and theoretical values of $\mu_{N}(B)$ $-\mu_{1}(0)$ to be compared provided that a model for $\Delta \mu_{c}(B)$ is available. A model investigated in the present work is $\Delta \mu_{c}(B)=\sqrt{\Gamma^{2}+\left(\hbar \omega_{c} / 2\right)^{2}}-\Gamma$, where $\Gamma$ is a fitting parameter. An advantage of analyzing chemical potential data is that Eq. (13) is less sensitive to errors in $\alpha$ than Eq. (11) however the disadvantage is that the results depend on the model chosen for $\Delta \mu_{c}(B)$.

Chemical potential data analysis requires a data set that contains $V_{g 1}$ so that $\mu_{N}(B)-\mu_{1}(0)$ can be found. However set 1 does not contain results for $N=1$ so set 2 is used instead.

The comparison between experimental and theoretical chemical potentials is shown in Fig. 5. As with the addition energy, a very good fit is obtained without any scaling or shifting of the theoretical data. The rms difference between the experimental and theoretical curves is about $0.12 \mathrm{meV}$. The best fit parameter values are $a=4.8 \pm 0.1 \mathrm{meV}$, $b=-0.06 \pm 0.02 \mathrm{meV}, \quad \lambda_{s}=16.0 \pm 2 \mathrm{~nm}, \quad$ and $\Gamma$ $=7.4 \pm 0.3 \mathrm{meV}$. Except for the sign of $b$, these values are consistent with the values obtained by fitting the addition
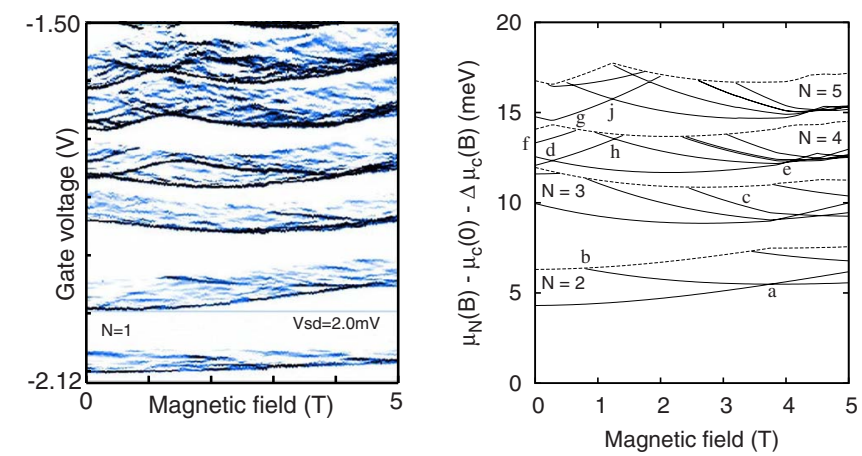

FIG. 6. (Color online) Experimental and theoretical excitation spectra. Left frame: experimental excitation spectra. The gray scale image shows $d I_{s d} / d V_{g}$ in the $B, V_{g}$ plane for $N=1-6$. Right frame: theoretical excitation spectra for $N=2-5$. The dashed lines show the upper bound of the excitation stripe, $\Delta E=2 \mathrm{meV}$. Roman font labels indicate the features listed in Table III.

energy. As with the addition energy the absolute magnitude of $b$ is small and the statistical error in $b$ is relatively large. This suggests that the variation in $\hbar \omega_{0}$ with $N$ is not very significant for the small $N$ range considered here.

The best model of the device is believed to be the model that results from fitting the addition energy because an extra parameter is needed to fit the chemical potential data and it is preferable to keep the number of fitting parameters to a minimum. The addition energy model was therefore used in Ref. 5 to determine ground-state quantum numbers from transport data. The visual comparison of excited state features in Ref. 5 was then performed with an approximate value of $\Gamma, 8.2$ $\mathrm{meV}$. This is valid in the case of the studies reported in Ref. 5 because the absolute value of $\mu=N(B)$ is not needed to identify the magnetic fields at which features occur.

\section{APPLICATIONS OF THE MODEL}

\section{A. Low field data}

The model parameters are found by fitting to the groundstate addition energies so comparison of theoretical and experimental excitation energies is a good test of the predictive power of the model. This comparison is performed here for the low magnetic field regime, $B \leq 5 \mathrm{~T}$, to which the model has not been applied before. A discussion of the high field regime is available in the literature, ${ }^{5}$ see also Sec. V B.

The experimental excitation energy data, Fig. 6 (left panel), consist of the derivative of the source-drain current, $d I_{s d} / d V_{g}$, plotted as an intensity image in the $B, V_{g}$ plane. The electron number ranges from 1 to 6 . The data were collected in a measurement separate from those leading to sets 1 and 2. The source-drain voltage $V_{s d}=2 \mathrm{mV}$ so the maximum excitation energy that can be probed is $2 \mathrm{meV}$. For each electron number there is a stripe that corresponds to this 2 $\mathrm{meV}$ window. The width of each stripe depends on $B$ and $V_{g}$ because of the $B$ and $V_{g}$ dependence of $\alpha$. Within each strip there are dark lines that correspond to transport through excited dot states. The excitation energy is found by scaling the line position to the stripe width. This gives excitation energies, $\Delta E$, to an accuracy of about $\pm 0.1 \mathrm{meV}$, together with 
field values accurate to about $\pm 0.02 \mathrm{~T}$. In addition to the excitation lines, there is "noise." Some of this is caused by emitter states which result from density of states fluctuations in the heavily doped contacts. To extract the $B$ dependence of the excitation lines it is necessary to distinguish contact features from dot features. This can be done by rejecting features that do not depend on $N$ (Ref. 5), for example. However the excitation lines in Fig. 6 have gaps and are of variable intensity so the present quantitative comparison is based on features such as level crossings and crossings of excitation lines with the upper boundaries of the excitation stripes. These features can be identified unambiguously by comparing the topology of the experimental and theoretical data.

The theoretical excitation energy data are shown in the right frame of Fig. 6. The figure shows $\mu_{N}(B)-\mu_{1}(0)$ $-\Delta \mu_{c}(B)$ for electron numbers in the range 2 to 5 . For each $N$ the energies of the ground state and lowest four excited states are calculated and the value of $\mu_{N}(B)-\mu_{1}(0)$ $-\Delta \mu_{c}(B)$ is found for the ground state and each of the excited states. The excited state lines that fit into the $2 \mathrm{meV}$ excitation window are shown by the solid lines in the figure. The continuous solid line at the bottom of each stripe is the ground-state chemical potential and this coincides with the lower boundary of each excitation window. The dashed line at the top of each stripe shows the upper boundary, $2 \mathrm{meV}$ above the ground-state line.

Qualitatively, there is very good agreement between the theory and experiment. However some of the experimental excited state lines are not continuous and there is some noise. The singlet-triplet transition of the two-electron system is clearly visible (a) and so is the excited state triplet line (b-a). For three electrons there are ground-state transitions in the 4-5 $\mathrm{T}$ interval in both the theory and experiment and an excited state line is visible in the experimental data. This may correspond to the second excited state line (c) in the theory. However it is difficult to identify this experimental line unambiguously because it does not extend to the excitation stripe boundary. It is also possible that this line corresponds to the first excited state line below line (c) in the theory. In the case of four electrons there are ground-state transitions (d) and (e) in both theory and experiment. In addition, an excited state line which has a flat maximum is present in the experimental data. This corresponds to the crossing (h) in the theory but small symmetry breaking effects such as disorder change the crossing into an anticrossing and lead to a maximum in the experimental data. The second excited state line (f-g) is also clearly visible in both the theory and experiment. For five electrons the groundstate transition $(j)$ has the distinctive form of a maximum and is clearly present in both theory and experiment, and some short excited state lines emerge from this crossing. Further, for both four and five electrons the experimental groundstate line is broadened in the regions between 4 and $5 \mathrm{~T}$. This corresponds to the region where the theory predicts that ground and excited state levels cluster together and excitation gaps shrink. Although the agreement is generally good, there are some discrepancies. For $N=1$ the theory predicts no excitations within the $2 \mathrm{meV}$ window at fields below $5 \mathrm{~T}$ but there is some structure in the experimental data. This is
TABLE III. Comparison of features in experimental and theoretical spectra. Features (a), (d), (e), and (j) are ground-state transitions. For these features $\Delta E=0$ and $(L, S) \rightarrow\left(L^{\prime}, S^{\prime}\right)$ indicates low field values of $(L, S)$ followed by high field values. Features (b), (f), $(\mathrm{g})$, and (h) are excitation energies at the points shown in Fig. 6 and defined in the text. For these features $(L, S) \Rightarrow\left(L^{\prime}, S^{\prime}\right)$ indicates excited state $(L, S)$ values followed by the ground-state values. The theoretical fields are accurate to $\pm 0.05 \mathrm{~T}$.

\begin{tabular}{|c|c|c|c|c|c|c|}
\hline Feature & $N$ & $\begin{array}{l}\Delta E_{\text {Exp }} \\
(\mathrm{meV})\end{array}$ & $\begin{array}{l}\Delta E_{\text {The }} \\
(\mathrm{meV})\end{array}$ & $\begin{array}{c}B_{\operatorname{Exp}} \\
(\mathrm{T})\end{array}$ & $\begin{array}{l}B_{\text {The }} \\
(\mathrm{T})\end{array}$ & $(L, S)$ values \\
\hline (a) & 2 & 0.0 & 0.0 & 4.2 & 3.8 & $(0,0) \rightarrow(1,1)$ \\
\hline (b) & 2 & 2.0 & 2.0 & 1.15 & 0.75 & $(1,1) \Rightarrow(0,0)$ \\
\hline (d) & 4 & 0.0 & 0.0 & 0.33 & 0.3 & $(0,1) \rightarrow(2,0)$ \\
\hline (e) & 4 & 0.0 & 0.0 & 4.06 & 4.0 & $(2,0) \rightarrow(3,1)$ \\
\hline (f) & 4 & 1.0 & 1.24 & 0.0 & 0.0 & $(0,0) \Rightarrow(0,1)$ \\
\hline (g) & 4 & 2.0 & 2.0 & 0.86 & 0.70 & $(0,0) \Rightarrow(2,0)$ \\
\hline (h) & 4 & 1.5 & 1.71 & 1.57 & 1.3 & $(0,1) \Rightarrow(2,0)$ \\
\hline (j) & 5 & 0.0 & 0.0 & 1.39 & 1.25 & $\left(1, \frac{1}{2}\right) \rightarrow\left(4, \frac{1}{2}\right)$ \\
\hline
\end{tabular}

thought to be caused by emitter states. Similarly, for $N=5$ no excitations are predicted in the $2 \mathrm{meV}$ window at zero magnetic field but there is some structure in the data.

The quantitative experiment-theory comparison for the features that can be identified unambiguously is detailed in Table III. In the case of the singlet-triplet transition of the two-electron system (a), the predicted transition field is about $0.4 \mathrm{~T}$ lower than in the experiment and the point where the triplet excitation line crosses the $2 \mathrm{meV}$ excitation boundary (b) is also about $0.4 \mathrm{~T}$ lower. This discrepancy is thought to be a consequence of the thermal cycling since the positions of the singlet-triplet transition agree well in the addition energy data, Fig. 4.

The four-electron system is particularly rich in features that can be identified unambiguously. Ground-state transitions at 0.33 and $4.06 \mathrm{~T}$ agree very well with transitions at $0.3 \mathrm{~T}(\mathrm{~d})$ and $4.0 \mathrm{~T}(\mathrm{e})$ in the theory. The excitation energy of the second excited state (f) at zero magnetic field agrees with theory to $0.24 \mathrm{meV}$ while the position of the crossing of the second excited state line with the excitation stripe boundary $(\mathrm{g})$ agrees with theory to $0.16 \mathrm{~T}$. The excitation energy at the excited state crossing (h) agrees to $0.21 \mathrm{meV}$ and its position agrees to $0.27 \mathrm{~T}$. It is more difficult to identify features in the five-electron data as there is more noise. However the position of the ground-state transition $(\mathrm{j})$ agrees to $0.14 \mathrm{~T}$.

\section{B. High field data}

Data in the high magnetic-field regime beyond the MDD is analyzed with the present model in Ref. 5. Although fitting procedures are not discussed in Ref. 5, it is emphasized that identical procedures and parameters are used in both Ref. 5 and the present work. One of the main findings of Ref. 5 is the existence of intermediate spin states in the regime beyond the MDD. The MDD is spin polarized but as the magnetic field increases a transition to a partly polarized state occurs, followed by a spin polarized state, then another 
partly polarized state and so on. The quantum numbers found for the $N=5$ dot studied in Ref. 5 are consistent with general ideas about symmetry and electron molecular states. ${ }^{7,8}$ In the specific case of the $N=5$ partly polarized states, calculations of the pair correlation function indicate that there is a superposition of four- and fivefold symmetry with a dominant fivefold component. Further details are given in Refs. 27 and 28.

\section{CONCLUSION}

An accurate model of a vertical pillar quantum dot has been developed which is able to reproduce addition energy and chemical potential data for individual quantum dots. Experimental addition energies are reproduced to an accuracy of around $0.15 \mathrm{meV}$ and this is accurate enough to enable ground-state spin and orbital angular momentum quantum numbers to be determined from transport data. Although the three-dimensional device structure is accounted for, the resulting physical model reduces to one in which electron motion is restricted to the two lateral dimensions, the confinement is parabolic and the interaction potential differs significantly from the bare Coulomb potential.

The model only contains three adjustable parameters. Of these the parameter that describes the $N$ dependence of the confinement energy does not appear to be significant over the small $N$ range considered here and it is likely that a two parameter model would give similar accuracy to the present one. For dots with larger $N$ it would be possible to include nonparabolic confinement. This would increase the number of model parameters but with larger $N$ there would be more data so the additional parameters could probably be determined reliably.

Although the model parameters are determined by fitting ground-state data, the model is able to give a good description of the low lying excited states. The model energies agree with experiment to about $10-20 \%$ and the positions of features in magnetic-field-dependent data agree to similar accuracy. One of the factors limiting the accuracy of the present analysis is uncertainty in the value of the electrostatic leverage factor $\alpha$. Accurate measurements of $\alpha$ would enhance the scope and applicability of the present analysis.

The general approach described here is not limited to vertical pillar dots. In principle, similar analysis procedures could be developed for any type of dot, provided a good physical model is available.

\section{ACKNOWLEDGMENTS}

S.T. is grateful for financial support from the Grant-in-Aid for Scientific Research CS (Grant No. 19104007) and B (Grant No. 18340081), SORST-JST and Special Coordination Funds for Promoting Science and Technology, MEXT, Japan. P.A.M. is grateful for hospitality at the Department of Physics, University of Tokyo where this paper was completed and is also grateful for special study leave from the University of Leicester. We are pleased to thank H. Imamura for fruitful comments and discussions.

\section{APPENDIX: NUMERICAL CALCULATION OF GREEN'S FUNCTION}

The functions $f$ and $g$ and the Wronskian $W$ [Eq. (7)] are needed to find the Green's function. To find $f$ and $g$ the system is divided into thin slices, such that $\epsilon$ and $q_{0}$ are constant in each slice. In the present case this simply means that each layer of the structure is subdivided into thin slices but the same numerical procedure is applicable to any form of $\epsilon(z)$. The $n$th slice occupies the region between $z_{n-1}$ and $z_{n}$ and is characterized by a relative permittivity $\epsilon_{n}, q$ value $q_{n}^{2}=q^{2}+q_{0}^{2}(z)$ and a thickness $t_{n}=z_{n}-z_{n-1}$. Within each slice $f$ and $g$ have the form $A_{n} \exp \left(-q_{n} z\right)+B_{n} \exp \left(q_{n} z\right)$. The two terms in this expression are analogous to evanescent waves in diffraction theory and it is convenient to use the language of diffraction theory to describe them. Thus the reflected wave $R$ which decays in the positive $z$ direction has the form $R=A_{n} \exp \left(-q_{n} z\right)$. Similarly, the transmitted wave $T$ which decays in the negative $z$ direction has the form $T$ $=B_{n} \exp \left(q_{n} z\right)$.

The amplitudes at successive slices are related via a transfer matrix,

$$
\left(\begin{array}{c}
R_{n+1} \\
T_{n+1}
\end{array}\right)=\left(\begin{array}{ll}
m_{1} & m_{2} \\
m_{3} & m_{4}
\end{array}\right)\left(\begin{array}{l}
R_{n} \\
T_{n}
\end{array}\right),
$$

where $R_{n}$ and $T_{n}$ are amplitudes just below the interface at $z_{n}$, that is within the slice of relative permittivity $\epsilon_{n}$. The boundary conditions on $G$ are used to find the elements of the transfer matrix: $g$ and $\epsilon(z) d g / d z$ are continuous and the same holds for $f$. Therefore

$$
\begin{gathered}
m_{1}=\frac{1}{2}\left(1+\frac{\epsilon_{n} q_{n}}{\epsilon_{n+1} q_{n+1}}\right) \exp \left(-q_{n+1} t_{n+1}\right), \\
m_{2}=\frac{1}{2}\left(1-\frac{\epsilon_{n} q_{n}}{\epsilon_{n+1} q_{n+1}}\right) \exp \left(-q_{n+1} t_{n+1}\right), \\
m_{3}=\frac{1}{2}\left(1-\frac{\epsilon_{n} q_{n}}{\epsilon_{n+1} q_{n+1}}\right) \exp \left(q_{n+1} t_{n+1}\right), \\
m_{4}=\frac{1}{2}\left(1+\frac{\epsilon_{n} q_{n}}{\epsilon_{n+1} q_{n+1}}\right) \exp \left(q_{n+1} t_{n+1}\right) .
\end{gathered}
$$

It is well known that direct application of Eq. (A1) is numerically unstable. Instead it is necessary to compute the ratio $r_{n}=R_{n} / T_{n}$ which corresponds to the reflection coefficient at each interface. ${ }^{21-23}$ The notation used here is similar to that of Ref. 23. The reflection coefficient $r_{n}$ satisfies

$$
r_{n+1}=\frac{m_{1} r_{n}+m_{2}}{m_{3} r_{n}+m_{4}},
$$

where the $m_{i}$ are the elements of the transfer matrix [Eq. (A2)] for going across the interface at $z_{n}$ to the interface at $z_{n+1}$. Equation (A3) can be used to step $r_{n}$ provided that the denominator of the fraction is not small. If the denominator does become small an alternative relation can be used to step $r_{n}^{-1}$ (Ref. 21) but this was not needed for the present work.

The procedure for finding $g$ is as follows. Deep inside the bottom contact $g$ decays exponentially so the only compo- 
nent present is $T_{n}$. Hence $r_{n}$ is found from Eq. (A3) starting from $r_{0}=0$. Once $r_{n}$ is known $g_{n}$ is found from the relations

$$
\begin{gathered}
T_{n}=\left(m_{3} r_{n}+m_{4}\right)^{-1} T_{n+1}, \\
g_{n}=R_{n}+T_{n}=\left(1+r_{n}\right) T_{n},
\end{gathered}
$$

which follow from Eq. (A1) and the definitions of $R_{n}$ and $T_{n}$. Equation (A4) is applied with the initial condition $T_{N}=1$, where the topmost slice ends at $z_{N}$. This procedure corresponds directly to the one used in diffraction theory.

The procedure used to find $f$ is slightly different. $f$ is required to decay exponentially deep inside the top contact, that is, it only has a reflected component there. This means that the inverse reflection coefficient $T_{n} / R_{n}$ vanishes deep inside the top contact. Hence it is convenient to re-express Eqs. (A3) and (A4) in terms of the inverse transfer matrix defined by

$$
\left(\begin{array}{c}
R_{n} \\
T_{n}
\end{array}\right)=\left(\begin{array}{ll}
n_{1} & n_{2} \\
n_{3} & n_{4}
\end{array}\right)\left(\begin{array}{c}
R_{n+1} \\
T_{n+1}
\end{array}\right),
$$

where $R_{n}$ and $T_{n}$ are amplitudes just below the interface at $z_{n-1}$, again within the slice of relative permittivity $\epsilon_{n}$. The boundary conditions on $G$ lead to expressions for the inverse transfer matrix elements:

$$
\begin{aligned}
& n_{1}=\frac{1}{2}\left(1+\frac{\epsilon_{n+1} q_{n+1}}{\epsilon_{n} q_{n}}\right) \exp \left(q_{n+1} t_{n+1}\right), \\
& n_{2}=\frac{1}{2}\left(1-\frac{\epsilon_{n+1} q_{n+1}}{\epsilon_{n} q_{n}}\right) \exp \left(-q_{n+1} t_{n+1}\right), \\
& n_{3}=\frac{1}{2}\left(1-\frac{\epsilon_{n+1} q_{n+1}}{\epsilon_{n} q_{n}}\right) \exp \left(q_{n+1} t_{n+1}\right),
\end{aligned}
$$

$$
n_{4}=\frac{1}{2}\left(1+\frac{\epsilon_{n+1} q_{n+1}}{\epsilon_{n} q_{n}}\right) \exp \left(-q_{n+1} t_{n+1}\right) .
$$

The inverse reflection coefficient satisfies

$$
\widetilde{r}_{n}^{1}=\frac{n_{3}+n_{4} \widetilde{r}_{n+1}^{-1}}{n_{1}+n_{2} \widetilde{r}_{n+1}^{-1}}
$$

and this relation is used to step $\widetilde{r}_{n}^{-1}$, starting from the initial condition $\widetilde{r}_{N+1}^{-1}=0$. Here the notation $\widetilde{r}$ is used to emphasize that $r$ and $\tilde{r}$ are computed with different initial conditions so $\widetilde{r}^{-1}$ is not the inverse of $r$. Once $\widetilde{r}_{n}^{-1}$ is known, $f_{n}$ is found from the relations

$$
\begin{aligned}
& R_{n+1}=\left(n_{1}+n_{2} \widetilde{r}_{n+1}^{-1}\right)^{-1} R_{n}, \\
& f_{n}=R_{n}+T_{n}=\left(1+\widetilde{r}_{n}^{-1}\right) R_{n},
\end{aligned}
$$

with the initial condition $R_{0}=1$.

The Wronskian $W$ is independent of $z$ and can be evaluated at any convenient position. This requires $f, g$ and their derivatives. The derivatives are found from

$$
\frac{d f}{d z}=q_{n}\left[-A_{n} \exp \left(-q_{n} z\right)+B_{n} \exp \left(q_{n} z\right)\right]=q_{n}(T-R)
$$

together with a similar expression for $d g / d z$. This leads to

$$
W=-2 q \epsilon_{w} \epsilon_{0} \frac{\left(1-\widetilde{r}_{n}^{-1} r_{n}\right)}{\left(1+\widetilde{r}_{n}^{-1}\right)\left(1+r_{n}\right)} f_{n} g_{n},
$$

which is valid away from dielectric interfaces.

The relations given here have been found to be numerically stable for the device considered in the present work. But in general it is possible for the calculation of the Wronskian to underflow, causing overflow in the calculation of the Green's function. However this is only likely to be a problem for very thick systems and where underflow could result from the exponential form of $f$ and $g$.
${ }^{1}$ S. Tarucha, D. G. Austing, T. Honda, R. J. van der Hage, and L. P. Kouwenhoven, Phys. Rev. Lett. 77, 3613 (1996).

${ }^{2}$ L. P. Kouwenhoven, T. H. Oosterkamp, M. W. S. Danoesastro, M. Eto, D. G. Austing, T. Honda, and S. Tarucha, Science 278, 1788 (1997).

${ }^{3}$ T. H. Oosterkamp, J. W. Janssen, L. P. Kouwenhoven, D. G. Austing, T. Honda, and S. Tarucha, Phys. Rev. Lett. 82, 2931 (1999).

${ }^{4}$ L. P. Kouwenhoven, D. G. Austing, and S. Tarucha, Rep. Prog. Phys. 64, 701 (2001).

${ }^{5}$ Y. Nishi, P. A. Maksym, D. G. Austing, T. Hatano, L. P. Kouwenhoven, H. Aoki, and S. Tarucha, Phys. Rev. B 74, 033306 (2006).

${ }^{6}$ P. A. Maksym and T. Chakraborty, Phys. Rev. Lett. 65, 108 (1990).

${ }^{7}$ P. A. Maksym, Phys. Rev. B 53, 10871 (1996).

${ }^{8}$ P. A. Maksym, H. Imamura, G. P. Mallon, and H. Aoki, J. Phys.: Condens. Matter 12, R299 (2000).
${ }^{9}$ C. Yannouleas and U. Landman, Rep. Prog. Phys. 70, 2067 (2007).

${ }^{10} \mathrm{~T}$. Chakraborty, Quantum Dots: A Survey of the Properties of Artificial Atoms (Elsevier, Amsterdam, 1999).

${ }^{11}$ S. M. Reimann and M. Manninen, Rev. Mod. Phys. 74, 1283 (2002).

${ }^{12}$ A. Kumar, S. E. Laux, and F. Stern, Phys. Rev. B 42, 5166 (1990).

${ }^{13}$ P. Matagne, J.-P. Leburton, D. G. Austing, and S. Tarucha, Phys. Rev. B 65, 085325 (2002).

${ }^{14}$ S. Bednarek, B. Szafran, K. Lis, and J. Adamowski, Phys. Rev. B 68, 155333 (2003).

${ }^{15}$ D. V. Melnikov, P. Matagne, J.-P. Leburton, D. G. Austing, G. Yu, S. Tarucha, J. Fettig, and N. Sobh, Phys. Rev. B 72, 085331 (2005).

${ }^{16}$ D. V. Melnikov, T. Fujisawa, D. G. Austing, S. Tarucha, and J.-P. Leburton, Phys. Rev. B 77, 165340 (2008).

${ }^{17}$ N. A. Bruce and P. A. Maksym, Phys. Rev. B 61, 4718 (2000). 
${ }^{18}$ D. Chaney and P. A. Maksym, Phys. Rev. B 75, 035323 (2007).

${ }^{19}$ G. Lommer, F. Malcher, and U. Rössler, Phys. Rev. B 32, 6965 (1985).

${ }^{20}$ L. D. Hallam, J. Weis, and P. A. Maksym, Phys. Rev. B 53, 1452 (1996).

${ }^{21}$ G. Meyer-Ehmsen, Surf. Sci. 219, 177 (1989).

${ }^{22}$ T. C. Zhao and S. Y. Tong, Phys. Rev. B 47, 3923 (1993).

${ }^{23}$ T. Kawamura and P. A. Maksym, Surf. Sci. 601, 822 (2007).

${ }^{24}$ T. Ando, A. B. Fowler, and F. Stern, Rev. Mod. Phys. 54, 437 (1982).
${ }^{25}$ W. R. Smythe, Static and Dynamic Electricity (McGraw-Hill, New York, 1968).

${ }^{26}$ W. H. Press, B. P. Flannery, S. A. Teukolsky, and W. T. Vetterling, Numerical Recipes in $C$ (Cambridge University Press, Cambridge, 1993).

${ }^{27}$ P. A. Maksym and H. Aoki, Phys. Status Solidi C 3, 3798 (2006).

${ }^{28}$ P. A. Maksym, R. Arita, and H. Aoki, Int. J. Mod. Phys. B 21, 1643 (2007). 